\title{
Study on the Influencing Factors of Family Education Investment Difference
}

\author{
Liu $\mathrm{Yu}^{1}{ }^{*}$ Zhang Wenjing ${ }^{2}$ \\ ${ }^{1}$ Institute of Political Science and Law, University of Jinan, Jinan, Shandong, China \\ ${ }^{2}$ Research Office, Women's Development Research Center of Shandong Province, Jinan, Shandong, China \\ •Corresponding author.Email: 1603491390@qq.com
}

\begin{abstract}
Combing the literature about the family education investment in recent years, the summary description and analysis of the academic circles on the current status of the family education investment, understand the existing in the study that the factors that influence the difference of family education investment to improve the rationality of the family education investment behavior, promote the rational allocation of education resources to provide related research foundation, for the follow-up related research ideas and directions.
\end{abstract}

Keywords: Family education investment, Family class, Educational resources

\section{RESEARCH BACKGROUND}

Education is the main way for individuals to improve their self-awareness level, and the key to the upward mobility of the disadvantaged and the change of their social status and destiny. Family education investment has always been an important way to affect children's education acquisition and ability development. With the continuous improvement of Chinese education system, the spread of rational education concept and the trend of family size as the core, more and more families pay attention to children's education investment. However, according to the existing research, the current family education investment is blind to a certain extent, and there are great differences in family education investment among different groups, which makes the impact of family education investment on children not play the best effect.

Therefore, on the basis of mastering the previous research on family education investment, this paper analyzes the current research results, summarizes the academic description of the current situation of family education investment, deeply studies the focus and direction of family education investment, and pays attention to the influencing factors leading to the differences in family education investment, so as to guide families to make rational education investment decisions To promote the efficient and reasonable allocation of educational resources, in order to provide ideas and directions for the follow-up research.
With the popularization of modern education concept and birth culture concept, there is almost no gender discrimination in education investment of children in poor families. However, the large number of children seriously weakens the ability of education investment of poor families. Moreover, the education investment of rural poor families lacks risk awareness and blindness, and does not realize that education investment is as risky as other investments, The cost-benefit analysis of education investment is also needed.

At present, the domestic and foreign research related to family education investment a lot, from the overall, the research focuses on the connotation of the family education investment classification, the current situation of the family education investment, the influence factors of the family education investment and education, family education investment results of internal mechanism and the countermeasure to improve the efficiency of the family education investment research these five aspects, This paper focuses on the first two aspects of the literature review.

\section{CURRENT SITUATION OF FAMILY EDUCATION INVESTMENT}

Academic research on the current situation of family education investment is very rich, and the research angle is very diverse. It mainly describes, compares and analyzes the current situation of family education investment around different types of families, different 
ways of family education investment and different motives of family education investment.

Family education investment is a special way of investment, which is fundamentally different from financial investment in nature, mode and time. The goal of financial investment is profit only, short-term benefit is pursued in time, and profit is the main investment; The education investment pursues the long-term goal of cultivating people. In addition to the capital investment, there is also the payment of knowledge and energy. It can be seen that parents' investment in children's education is huge, and according to the conclusion of this study, parents basically do not seek return. With the growth of children and the aging of parents, the problem of providing for the aged will become a reality. Pension is also a social concern, especially in rural areas.

\subsection{Different Types of Family Education Investment}

In the related research on family education investment, the research objects mainly include migrant children's families, rural families, urban families, poor families, families with minimum living allowance, families with special children, etc. among them, the most frequent research objects are rural families and urban families. Some scholars start from the perspective of rural families, This paper makes an empirical study on the influencing factors of rural poor families' willingness to invest in education; Some scholars have analyzed the current situation and characteristics of school education investment and out of school education investment of urban households in China. ${ }^{[1]}$

Some scholars have made a comparative analysis of the current situation of the differences between urban and rural family education investment, and concluded that the direction of rural education investment is single, while the direction of urban family education investment is diversified; The difference between urban and rural areas is mainly reflected in the investment cost of family education and out of school education. Rural parents spend little on family education and out of school education. The average investment cost of family education and out of school education of urban compulsory education students is significantly higher than that of rural students; At the stage of compulsory education, the education burden rate of single student's family in the city is much higher than that in the countryside, and the family education investment in the countryside is low.

\subsection{Family Education Investment in Different Ways of Investment}

Different ways of family education investment will also affect the final results and efficiency of education, so there are a lot of researches on family education investment. Some scholars explore the impact of economic investment and time investment in family education on adolescents' academic performance, and find that compared with economic investment, parents' time investment is the main factor affecting children's academic development. Some scholars believe that family emotional involvement has a significant impact on children's cognitive ability; Parents' financial support for their children's education has no significant positive effect on improving their children's cognitive ability. The research further shows that parents' emotional investment can affect the healthy development of children's cognitive ability more than all kinds of economic investment.

According to the relevant empirical research, in the family education investment, parents' emotional investment accounts for a higher proportion than time investment accounts for a higher proportion than economic investment, and the effect is better. Some scholars have studied the misunderstanding of family education investment behavior, and analyzed it from four aspects: paying attention to children's education investment; Neglect of energy and emotional input; Lack of rational thinking about children's situation and their own educational behavior, despise the cultivation of behavior habits and ideological and moral education.

With the increase of the number of children in rural families, it is more and more urgent for rural families to realize their children's desire to enter university and social mobility through education investment; In families with many children, influenced by traditional fertility culture, there are significant gender differences in children's educational expectations; However, families with few children, influenced by modern fertility culture, have no gender preference for children's educational expectations.

\subsection{Family Education Investment with Different Investment Motives}

In the research of family education investment on investment motivation, some scholars divide the concept of children's education investment of urban poor families into seven basic types, and think that the concept of children's education investment of urban poor families in China has the characteristics of gradual change from "laissez faire" to "meticulous cultivation", The concept of investment is characterized by passivity, blindness and fragmentation; The investment motivation is mainly academic performance type and skill development type, while the personality development type is insufficient; Investment expectation is generally low, and there is no clear plan for children's future development. [2]

Some studies have shown that the motivation of Chinese parents to invest in their children's education includes seven aspects. Among them, in order to fulfill 
their social responsibilities, parents' obligations and improve their children's survival ability, the three motivation needs are more prominent; In addition, parents in rural areas hope their children can feed back and fulfill their parents' wishes more than those in urban areas. Parents are driven by different educational investment motives, which makes them make different choices when making family educational investment decisions, and ultimately achieve different educational effects.

\section{INFLUENCING FACTORS OF FAMILY EDUCATION INVESTMENT DIFFERENCES}

The analysis of the factors affecting the investment of family education in academic circles is very rich. The analysis of the influencing factors in different disciplines and fields is involved. But the factors influencing the differences in family education investment mainly include family economic factors, parents' characteristics and family children's characteristics.

\subsection{The Influence of Family Economic Factors on Family Education Investment}

Some scholars have pointed out that the level of family income is an important factor affecting urban and rural residents' investment in children's education. The scale of education investment of low-income families in China is generally low, but the burden rate of education expenditure is higher than that of high-income families. The lower the income of urban and rural residents, the heavier the burden of education expenditure. Cardak believes that income inequality makes low-income families relatively lack of investment in children's education, and can not give their children more education investment, while high-income families can rely on their own economic resources and status, through the way of education rent-seeking, provide more education investment for their children, so that their children can get more high-quality education resources and opportunities. ${ }^{[3]}$ Therefore, the widening income gap between urban and rural areas and class will also aggravate the inequality of family education expenditure.

The higher the poor families' satisfaction with the education fiscal expenditure policy, the lower their willingness to invest in education, which shows that there is a trade-off relationship between the government's financial investment and the farmers' family education investment. The government's increasing financial investment will reduce the education burden of farmers' families, especially poor families, and help poor families realize the expected income of education investment. On the contrary, too small financial investment will increase the burden of education for farmers, which is not conducive to the realization of the goal of education development and the expected income of farmers' education investment at the same time.

Other scholars have conducted an investigation and empirical research on the investment in compulsory education of urban residents' families, and found that the greater the burden of compulsory education of urban residents, the lower the family income. However, the education burden of low-income families is greater than that of high-income families, and the education level of school students is directly proportional to the degree of family education burden. Some studies have found that the children of urban and rural families represented by household registration and different classes of families mainly represented by income have different educational opportunities and educational quality. The children of non-agricultural household registration and higher income families have significantly more educational investment than those of agricultural household registration and lower income families. The main reason behind this is the huge income gap between urban and rural residents. ${ }^{[4]}$

\subsection{The Influence of Parents' Characteristics on Family Education Investment}

The factors of parents' characteristics include parents' education concept, occupation type, parents' education level and so on. Research shows that parents with higher education usually send their children to kindergartens that meet their own expectations; However, parents with lower education can only send their children to kindergartens that they can afford. In addition, some scholars have also shown that parents' educational level is positively correlated with the scale of family investment in out of school education, and parents with higher political authority can reduce their children's school choice costs to a certain extent. Due to the imperfection of the education system, the families with higher professional power are rent-seeking for the power of high-quality education resources, so they can get better and cheaper education opportunities through less education investment. Highly educated parents usually send their children to kindergartens that meet their own expectations; Low educated parents usually send their children to kindergartens that they can afford.This kind of research shows that professional authority has economic compensation for education investment, which can reduce the higher class's education burden rate or improve the education return rate to a certain extent. 


\subsection{The Influence of Kids' Characteristics on Family Education Investment}

Culture is a very important factor affecting the motivation of family education investment. It needs to be analyzed from different hierarchical dimensions, we speculate that parents in individualistic culture are less worried about their children's future than those in collectivist countries, and their scores in social responsibility, parental obligations and traditional concepts are lower than those in collectivist countries.

Previous studies have shown that the gender, number and birth order of children affect the distribution of family education investment to a certain extent. Some studies divide children's educational resources into economic input, parents' participation and family environmental resources. It is found that although there are generally fewer siblings in China, the educational resources and academic achievements still decrease significantly with the increase of siblings. The investment of migrant children's family education to those who have brothers and sisters is less than that of the only child; Affected by the preference of boys, migrant families invest more in boys' education than girls'; The preference of migrant children families for birth order shows that they invest more in family education for boys born later. ${ }^{[5]}$ Research shows that the number of siblings, gender and birth order will dilute the family's educational resources to a certain extent. In contrast, the degree of dilution of money investment is smaller, the degree of dilution of time investment is larger, and the degree of dilution of goods investment is the largest.

\section{CONCLUSION}

With the continuous research on family education investment, the problems in the current situation of family education investment in our country have been paid more and more attention. However, it has not been paid much attention in China, and relevant studies are scarce in quantity. In recent years, as the problem becomes increasingly hot, domestic studies have begun to cover all aspects of family education investment: The connotation of the family education investment classification, the current situation of the family education investment, the influence factors of the family education investment and education, family education investment results of internal mechanism and the countermeasure to improve the efficiency of the family education investment research, for the adjustment of the family education investment gives many Suggestions, but the whole, there are three problems:

First, the current academic discussion on the influencing factors of family education investment is basically still at the level of theoretical discussion and empirical cognition, and there is a lack of empirical mechanism analysis driven by theory and data. In addition, the concept of family education investment is a multi-dimensional latent variable, and the traditional quantitative methods such as multiple regression analysis are not applicable to explore the causal relationship between latent variables. Therefore, it is necessary to adopt a new method to further explore the factors affecting family education investment.

Secondly, the existing literature on the factors affecting family education investment is still not deep enough, mostly at the why level, there is still a need to further explore the deep reasons behind the phenomenon, and in the future research needs to continue to find the factors affecting family education investment from the institutional and policy sources.

Thirdly, family educational investment and educational outcomes usually appear separately as research topics, and most of the studies are conducted from the surface characteristics and influencing factors of family educational investment. There are few studies on the influence mechanism of family educational investment on educational outcomes. Future research can explore the causal mechanism of the impact of family education investment on educational outcomes.

In view of the blindness in the current family education investment, the government should guide the poor rural families to objectively understand the educational investment and its existing risks, so that it can maximize the income of education investment combined with their own actual situation.

\section{REFERENCES}

[1] He Jianqing, $\mathrm{F}$ actors that affect poor rural house holds' willingness of investing in children's education, Education Research Monthly, 2014(03): 28-31.

DOI: https://doi.org/10.16477/Lcnki.issnl674-231 1.2014.03.002

[2] Wang Yuanwei, Zhu Sufei, The situations and characteristics of the educational inputs of Chinese urban families, Education \& Economy,2009(04): 11-16.

DOI: https://doi.org/CNKI:SUN:JYJI.0.2009-04-002

[3] Clarke, More Evidence on Income Distribution and Growth, Journal of Development Economics, 1995(2):403127.

DOI:

https://doi.org/10.1016/03043878(94)00069-O

[4] Jiang Yali, Teng Yun, On the upper mobility of the inferior class: A perspective of cultural reproduce of education, Journal of Guangzhou University (Social Science Edition), 2015(02): 45-51. 
DOI:https://doi.org/CNKI:SUN:GZDX.0.201502-00 8

[5] Liu Baozhong, "Widening Gap": the overall status and group differences of Chinese families' educational,Journal of Beijing University of Technology (Social Science Edition ) 2020(03): 1624.

DOI:https://doi.org/10.12120/bitskh20200216 